\title{
Faseroptische Bragg-Gitter-Sensorarrays zur Ausnutzungssteigerung großer Turbogeneratoren durch direkte Erfassung der Wicklungstemperatur auf Hochspannungspotential
}

\author{
S. Strack, J. R. Weidner, Siemens AG, Energy Sector, D-45478 Mülheim a. d. Ruhr \\ T. Bosselmann, M. Villnow, M. Willsch, Siemens AG, Corporate Technology, D-91058 Erlangen
}

\section{1) Einleitung}

Der stark wachsende Anteil erneuerbarer Energien an der Stromerzeugung erfordert eine höhere Flexibilität in der Betriebsweise konventioneller Kraftwerke. Zusammen mit dem verschärften Wettbewerb im liberalisierten Erzeugungsmarkt führt dies zu erheblich gestiegenen technischen Anforderungen an die modernen Kraftwerksblöcke und somit auch an deren Kernkomponente mit der größten Energiedichte, dem Turbogenerator. Für eine bessere Ausnutzung des Generators bei gleichzeitig hoher Verfügbarkeit und langer Lebensdauer ist das thermische Verhalten der Ständerwicklung von ausschlaggebender Bedeutung. Mit der genauen Kenntnis der maximalen Kupferleitertemperatur bei den verschiedenen Betriebszuständen ließe sich die thermische und elektrische Auslegung indirekt gekühlter Generatorständer optimieren [1]. Auch das thermische und thermo-mechanische Alterungsverhalten der Hochspannungsisolierung könnte durch eine direkte Messung der Kupferleitertemperatur über Jahrzehnte kontrolliert werden. Trotz hoher Ausnutzung der Ständerwicklungsisolierung wäre auch bei hohen dynamischen Betriebsbeanspruchungen eine Lebensdauer von über 30 Jahren möglich. Diesen Herausforderungen, die im Rahmen u.a. des geplanten massiven Einsatzes von „Erneuerbare Energien“ auf große Turbogeneratoren anstehen, könnte damit begegnet werden

Der direkten Überwachung der Kupferleitertemperaturen durch integrierte Temperatursensoren kommt somit eine besondere Bedeutung zu. Da sich die Kupferleiter der Ständerwicklungen bei Betrieb auf Hochspannungspotential befinden, sind metallische Temperatursensoren nicht geeignet, da sie zu Kurzschlüssen führen würden. Zudem wären die metallischen Sensoren und Messleitungen durch die hohe Energiedichte bei Generatoren sehr starken elektrischen und magnetischen Störfeldern ausgesetzt.

Technologien, die auf optischen Funktionsprinzipien beruhen, spielen bei der Temperaturerfassung sowie der Schwingungsmessung eine Schlüsselrolle [2]. Am Beispiel des Prototypentests der neuen luftgekühlten 370MVA Klasse von Turbogeneratoren der Siemens AG wird der Einsatz von „Faser-BraggGitter" (FBG) Temperatursensoren an der 18kV Generatorständerwicklung vorgestellt. Ebenso wird die Bedeutung von optischen Schwingungssensoren und die erweiterten Analysemöglichkeiten, die diese in Verbindung mit Kenntnis der Ständerkupferstabtemperatur bieten, vorgestellt.

Der getestete Turbogenerator mit einer Darstellung von Art, Anzahl und Messort der verwendeten Sensoren ist in Bild $1.1 \mathrm{zu}$ sehen. In dieser Abhandlung soll der Schwerpunkt auf die optischen Temperaturund Schwingungssensoren gelegt werden, weil diese für die Analyse des Betriebsverhaltens von großen Turbogeneratoren entscheidend sind. 


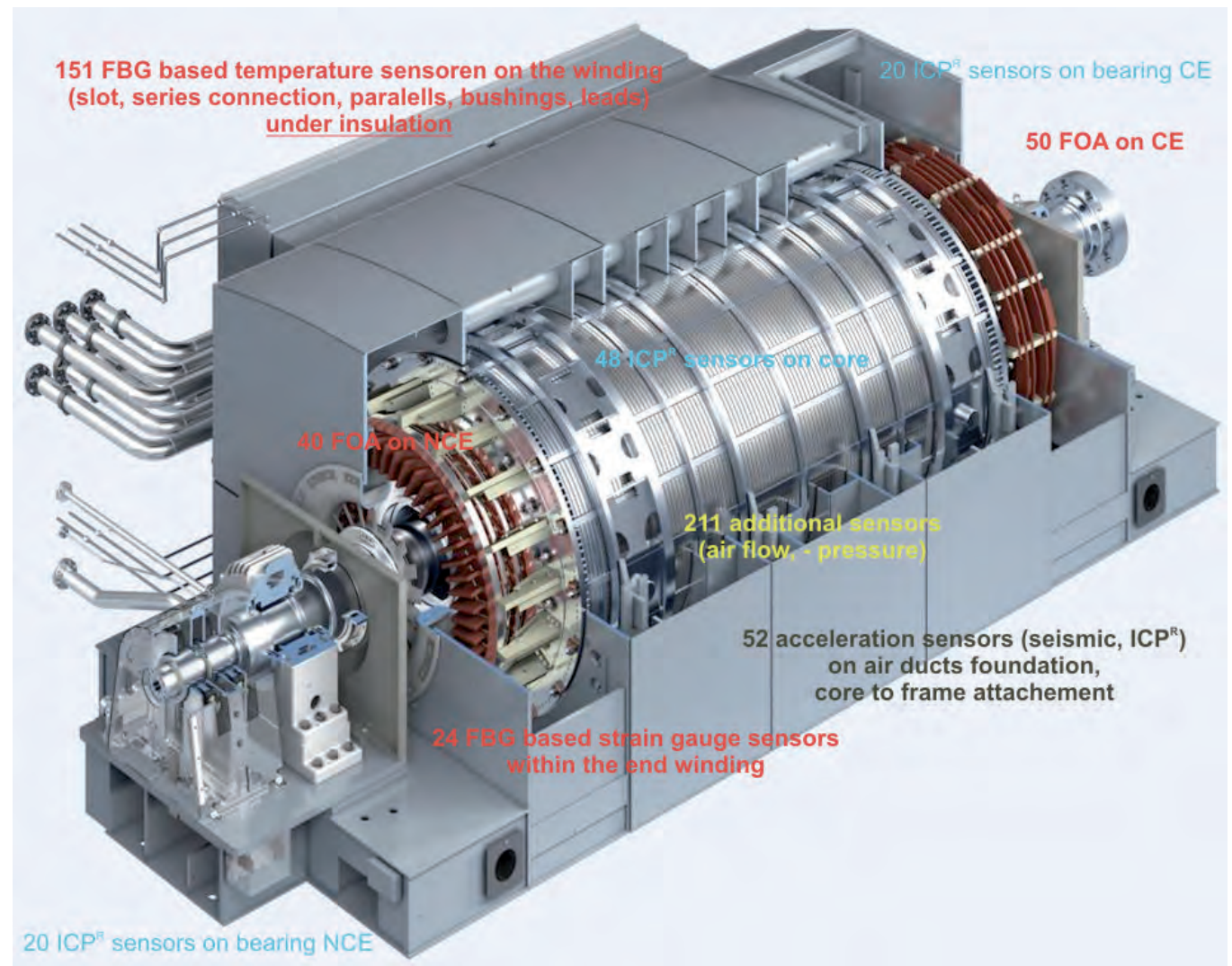

Bild 1.1: Luftgekühlter Turbogenerator mit Sensorpositionen und -anzahl

\section{2) Funktionsweise Faser-Bragg-Gitter für Temperaturmessung}

Für den Prototypentest ergaben sich verschiedene Messaufgaben zur Erfassung der Temperatur. Zum einen sollten Einzelsensoren die Temperaturen an ausgewählten Punkten der Aus- und Verbindungsleitungen, welche aus massiven Kupferschienen bestanden, überwachen. Zum anderen sollte an mehreren Positionen entlang der Generatorwicklung unterhalb der Isolierung die Temperaturverteilung ermittelt werden. Um eine möglichst einheitliche Messtechnologie zu gewährleisten, wurden alle Temperatursensoren auf der Basis der Faser-Bragg-Gitter FBG Technologie aufgebaut. Die FBG Technologie ist mittlerweile in der optischen Telekommunikationstechnik weit verbreitet, und es gibt auch einige Hersteller kommerzieller Sensorsysteme basierend auf dieser Technologie. Für die Messung der Temperatur in der Generatorwicklung bestand die Aufgabe, entsprechende Gehäuse für die Einzelsensoren als auch für die Sensorketten zu entwickeln. Vorbedingung war, dass die gehäusten Sensoren und deren Einbau den Fertigungsprozess nicht behindern und die Isolationseigenschaften nicht herabsetzen. Sie mussten robust genug aufgebaut sein, um nach Durchlauf des gesamten Fertigungsprozess noch einwandfrei zu funktionieren. Ein Ersetzen fehlerhafter Sensoren nach Fertigungsende war nicht möglich. Mit der FBG Technologie war es möglich, Einzelsensoren als auch Sensorketten zu fertigen. Letztere konnten vorteilhaft auf den Generatorwicklungsstäben eingesetzt werden. Auf einem Lichtwellenleiter LWL waren bis zu 12 FBG eingeschrieben, wobei die Position der Sensoren durch vorher durchgeführte Modellrechnungen festgelegt wurde.

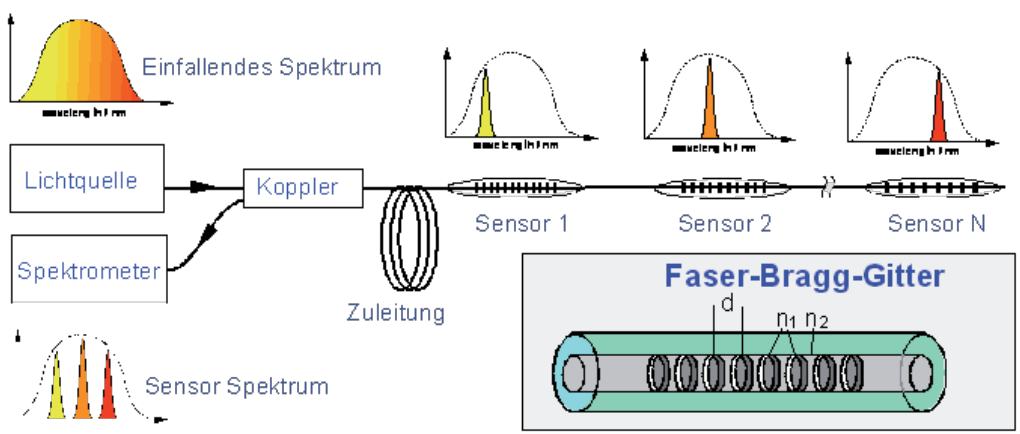

Bild 2.1: Wirkungsweise von Sensor-Arrays mit Faser-Bragg-Gittern (FBG) 
Die Multiplexing Möglichkeit erlaubte das gleichzeitige Auslesen der Temperaturmesswerte in einem Strang über eine einzelnen LWL. Dies war besonders vorteilhaft, als nur ein LWL durch die Wicklungsisolierung hindurchgeführt werden musste. Das Risiko der Herabsetzung der Isolationsfestigkeit wurde so minimiert. Durch eine speziell entwickelte Verlegetechnik konnte sichergestellt werden, dass die Isolationsfestigkeit erhalten blieb. Die FBG Sensoren funktionieren nur dann als Temperatursensoren einwandfrei, wenn sie spannungsfrei gelagert werden. Um dies unter der Isolierung der Wicklung zu gewährleisten, wurden die Sensorketten in passende dünne Quarzkapillaren eingeführt. Ein Ende wurde bündig verschlossen. Die Sensorkette wurde so positioniert, dass sie unter keinen Umständen an dieses Ende stoßen konnte. Die Zuleitung der Sensorkette wurde aus dem anderen Ende der Kapillare herausgeführt und dieses ebenfalls hermetisch verschlossen. Dadurch wurde sichergestellt, dass während der Einbettung des Sensors und der weiteren Fertigungsschritte kein Fremdmaterial in die Kapillare eindringen konnte.

Die Einzelsensoren sollten teilweise die Temperatur im Kupferleiter messen. Daher wurden die Einzelsensoren ähnlich aufgebaut wie die Ketten. Ein dünnes Röhrchen, diesmal aus Keramik, wurde an einem Ende wieder bündig verschlossen. Beim Einzelsensor saß das Gitter so nah wie möglich am LWL Ende, typ. wenige $\mathrm{mm}$. Der LWL mit dem FBG wurde gerade soweit hineingeschoben, dass er nicht am anderen Ende anstieß. Das andere Ende wurde darauf versiegelt und damit die Position des LWL fixiert. Da für die Zuleitung keine aufwändige Verlegetechnik durch die Isolierung notwendig war, konnte der LWL als Faser-Ader mit entsprechender Teflonhülle weitergeführt werden. Dies erhöhte die Robustheit dieses Sensorkonzepts erheblich.

Die Sensoren wurden mit Hilfe einer Breitband LED und eines Miniaturspektrometers ausgelesen. Dies hatte den Vorteil, dass für alle Sensoren das gleiche Ausleseprinzip zum Einsatz kam. Zusätzlich war in die Auswerteeinheit AWE ein 1 auf 7 Faseroptischer Schalter integriert. Damit konnten nacheinander 7 Einzelsensoren bzw. Sensorketten ausgelesen werden. Die Umschaltzeit betrug wenige $10 \mathrm{~ms}$. Verglichen mit den relativ langsamen Temperaturänderungen bedingt durch die sehr großen Massen, kam dies quasi einer Simultanmessung gleich. Waren 7 Sensorketten mit jeweils 12 Sensoren angeschlossen, so konnten parallel 84 Temperaturmeßstellen ausgelesen werden. Neben den langen Sensorketten für den Geradteil der Wicklung wurden noch kurze Ketten mit 5 FBG gebaut. Diese hatten einen besonders flexiblen Aufbau, da sie auf den gebogenen Enden der Wicklung, den Evolventen appliziert wurden.

\section{3) Realisierung Prototypentest Turbogenerator}

Die Technologie der Temperaturmessung durch Faser-Bragg-Gitter auf Hochspannungspotential (18kV) wurde an dem neuen luftgekühlten Generatortyp der 370MVA Klasse eingesetzt.

Die Implementierung von FBG-Sensoren auf den Kupferleitern der Ständerwicklung diente folgenden Zielen:

- Verifizierung der komplexen Rechenmodelle für die Generatorauslegung, um durch eine genaue Kenntnis der Wicklungserwärmung bei verschiedenen Betriebszuständen zu erlangen und um eine hohe, aber dennoch dauerhaft betriebssichere Maschinenausnutzung zu erreichen.

- Online-Überwachung der maximalen Kupferleitertemperatur bei verschiedenen Betriebszuständen zwecks Vermeidung thermischer Schädigung der Isolierung.

Die Herausforderung bestand in der Adaption dieser Technologie im Rahmen des Fertigungsprozesses. Die Positionen der Temperaturmessung auf den Ständerwicklungsstäben wurden durch Ergebnisanalysen von Modellrechnungen ausgewählt und hinsichtlich der Umsetzbarkeit der Sensorenapplikation analysiert. Bild 3.1 zeigt die Messpositionen während der Abschlussschritte der Stabfertigung bei Siemens. Zur Anwendung kam hier eine Messkette mit verschiedenen Bragg-Gitter-Strukturen in einer Faser, die während des Fertigungsprozesses auf der Schmalseite des Stabes appliziert wurde. Ziel war die messtechnische Erfassung des Kupfertemperaturprofils innerhalb der Blechpaketnut. 

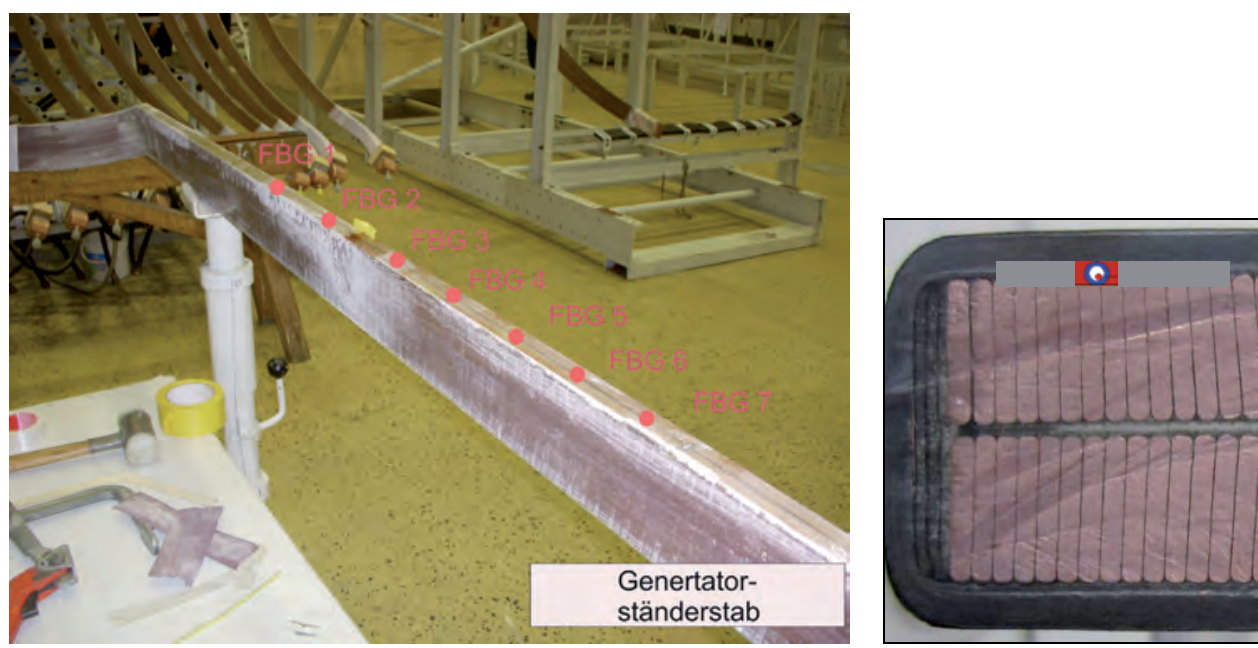

Bild 3.1: Ständerstab mit angedeuteten Positionen der Bragg-Gitter-Messstellen

In Bild 3.2 ist der applizierte Stab zu sehen, nachdem die Stabisolierung aufgebracht worden ist. Dabei wurden die durch die starken elektrischen Felder notwendigen Randbedingungen beachtet.

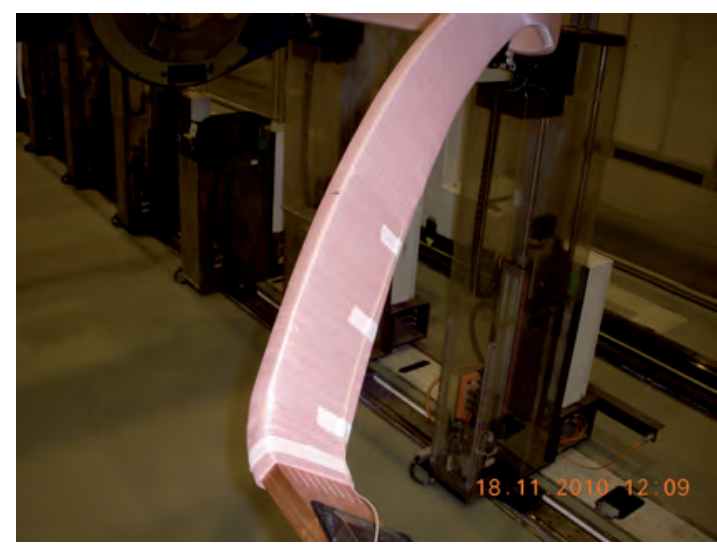

Bild 3.2: Ständerstab mit herausgeführter Bragg-Gitter-Messstellen-Zuleitung, nach dem Aufbringen der Isolierung, vor dem Isolierharz-Tränkprozess

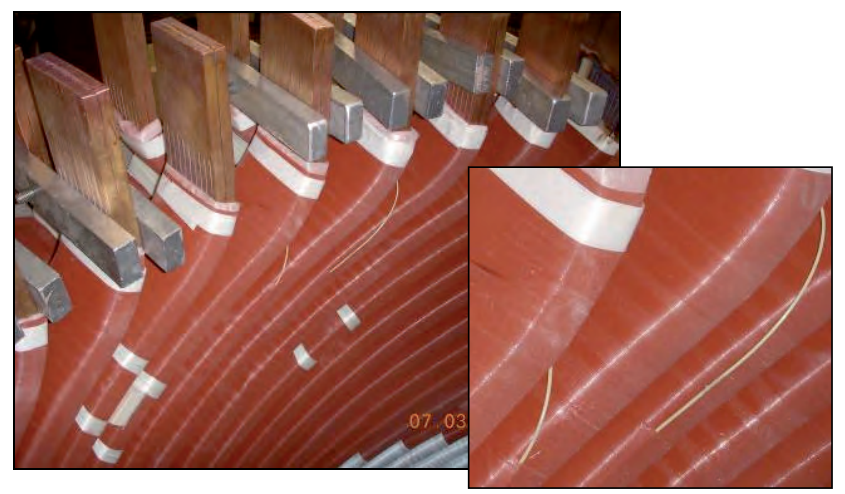

Bild 3.3: Austritt der Lichtwellenleiter aus der Hochspannungsisolierung im Ständerwickelkopf

Bild 3.3 und 3.4 zeigt die Generatorwicklung eingelegt ins Blechpaket mit den jeweiligen Messpositionen FBG x. Zusätzlich sind hier optische Schwingungssensoren FOA 1, FOA 2, zu erkennen, die auf dem Prinzip der Intensitätsmodulation beruhen. Der kombinierte Einsatz der verschiedenen optischen Sensortechnologien macht es möglich das Maschinenverhalten in Abhängigkeit unterschiedlicher Lastzustände exakt zu verifizieren. 


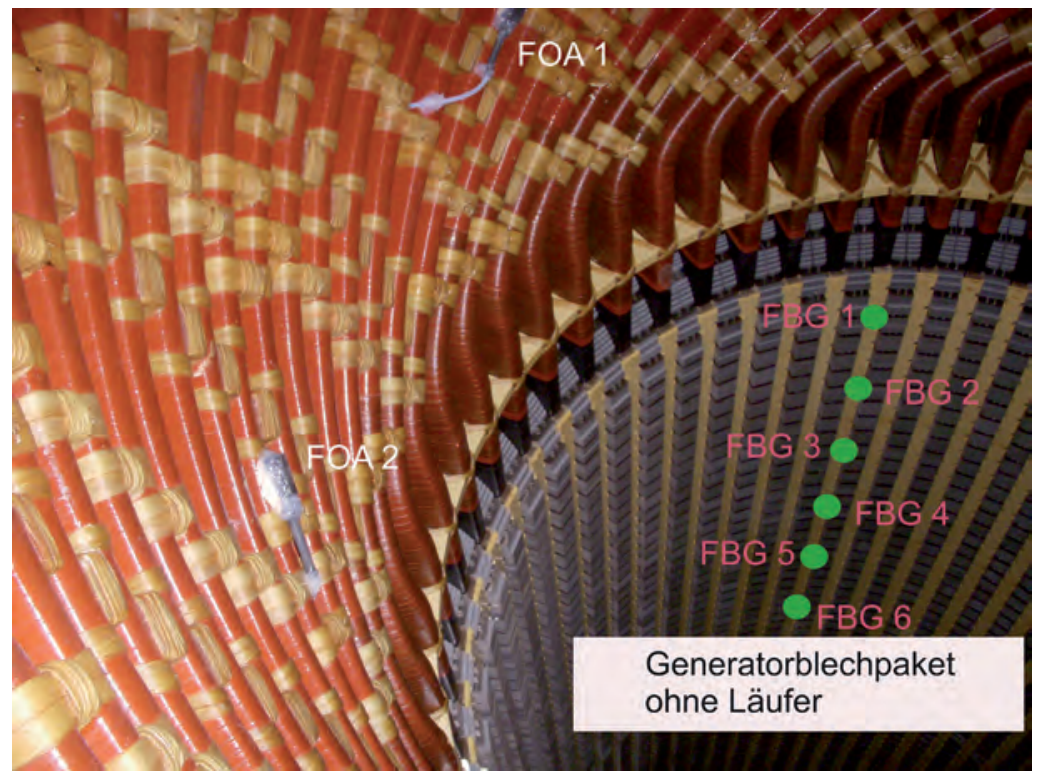

Bild 3.4: Fertiger Generatorständer ohne Rotor mit FBG- und FOA- (fiber-optic-accelerometer) Positionen

In Bilder 3.5 ist das Generatortestfeld im Werk Mülheim a. d. Ruhr der Siemens AG zu sehen. Links im Bild ist der Generator inklusive seines Luftkühlgehäuses zu sehen, rechts daneben Container, die das Equipment zur Messdatenerfassung und -speicherung beinhalten. Rechts im Bild kann man den Stator eines luftgekühlten Generators sehen, sowie dahinter die Antriebsmaschine für den Prüfstandslauf.

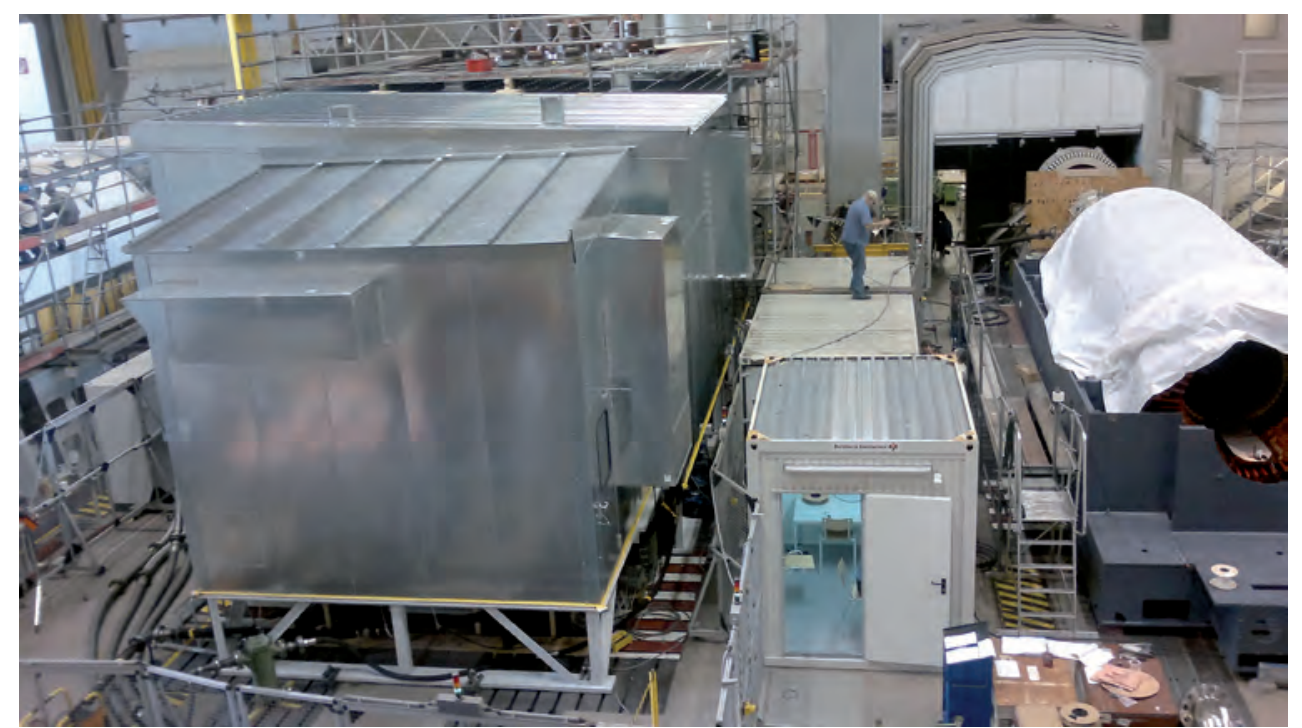

Bild 3.5: Generatortestfeld, Werk Muelheim an der Ruhr, Siemens AG

Das Bild 3.6 zeigt die optische Auswerteinheit „Slot" für die Temperatursensoren in der Wicklung entlang des Nutbereichs. Links an der Frontseite sind die Eingänge für den Faserschalter zu sehen. Die AWE steht auf einem Gehäuse mit 8 1x4 Verzweigern. Mithilfe dieser Faserkoppler konnten Einzelsensoren spektral gebündelt werden, um dann simultan ausgelesen werden zu können. 


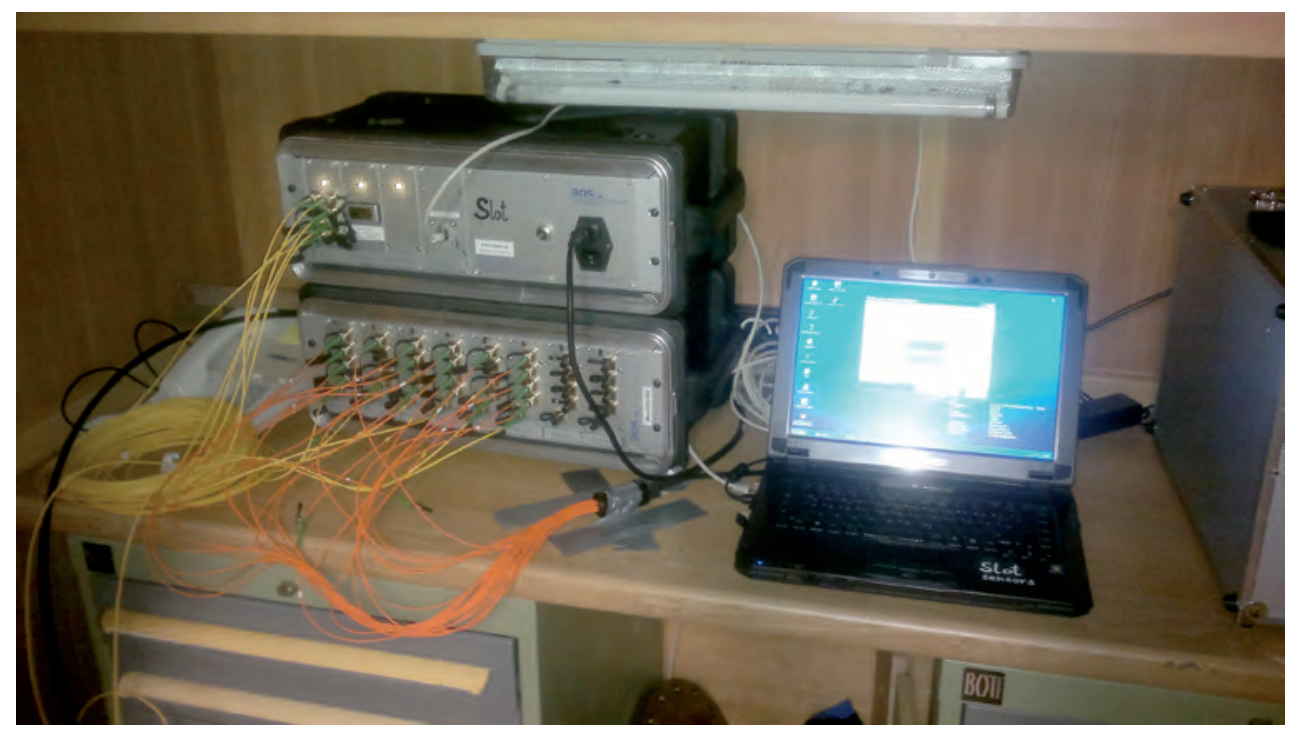

Bild 3.6: Auswerteeinheit (AWE) mit 1x7 Schalter und 1x4 optischem Verzweiger

Bild 3.7 zeigt Ergebnisse der Temperaturmessung in der Blechpaket-Nut des Generators in Korrelation zu parallel aufgenommenen Schwingungen am Blechpaket und im Wickelkopfbereich mit verschiedenen Lastzuständen. Die Erhöhung des Erregerstroms If verursacht die Erhöhung der Kupferstabtemperatur (FBG 1-30). Gleichzeitig ist die Erhöhung der Blechpaketschwingung zu erkennen und ein Absinken der Wickelkopfschwingung. Diese Effekte zeigen deutlich die Zusammenhänge zwischen Wicklungserwärmung und Schwingungsamplituden. Die geringen Änderungen in der Blechpaketschwingung zeigen, dass im Betriebsfall „Klemmen-Kurzschluss“ die verursachenden Effekte des elektrischen Stroms überwiegen. In der obersten Spur ist der zeitliche Temperaturverlauf zu sehen. Man erkennt deutlich den Anstieg Temperatur. Die zeitliche Aufwärmung zieht sich über Stunden hin aufgrund der großen Masse des Stators. In der Spur darunter ist ein Schwingungssignal zu sehen, erkennbar ist die Korrelation der Schwingungsamplitude mit der Temperatur, mit steigender Temperatur nimmt die Schwingungsamplitude ab. Während die Schwingungsamplitude es in der untersten Spur aufgezeichneten Sensors zunimmt.

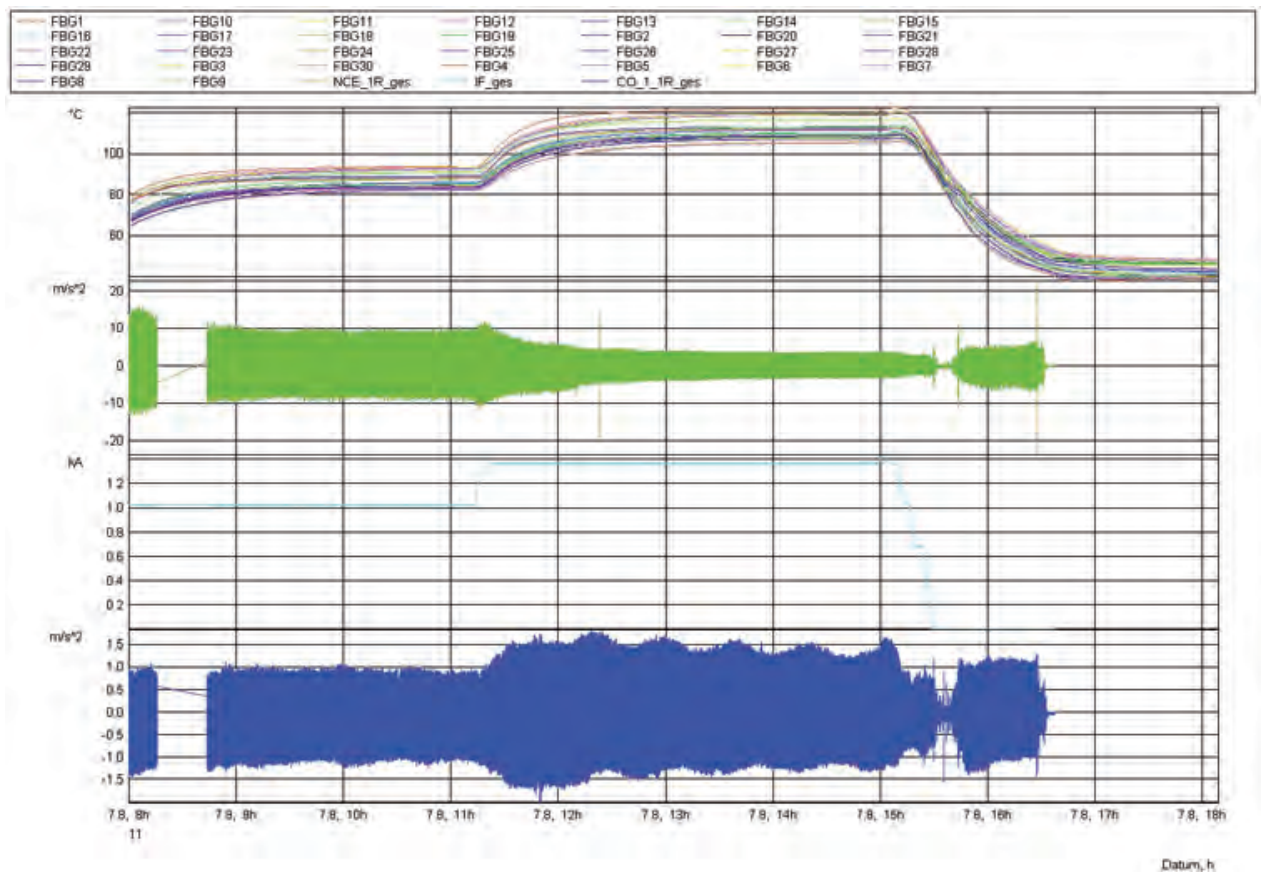

Bild 3.7: Fenster 1-FBG Temperaturen, Fenster 2-Schwingungen im Wickelkopfbereich, Fenster 3- Generatorerregerstrom, Fenster 4-Blechpaketschwingungen 
Bild 3.8 zeigt 4 FBG Temperatursensoren im örtlichen Verlauf an 4 unterschiedlichen Ständerstäben, welche durch die unterschiedliche Kühlzonenaufteilung zustande kam. Der wellenförmige Verlauf der Messkurven zeigt an, an welchen Positionen die Kühlschlitze die Wicklung kühlen (Temperatur-Tal), und welche Positionen im Windschatten liegen (Temperatur-Berg)

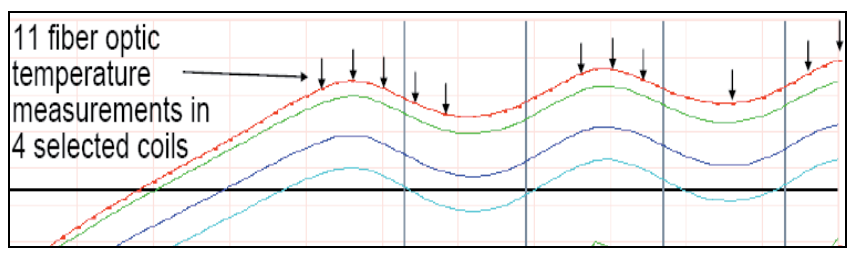

Bild 3.8: Faseroptische Temperaturmessung an vier ausgewählten Wicklungsstäben über der axialen Länge während des mechanischen Leerlaufs

In Bild 3.9 werden zusätzlich die Einflüsse der Drehzahl auf das Maschinenverhalten dargestellt. Erregerstrom und Phasenstrom sind direkt proportional zueinander, da der Generator im „Klemmenkurzschluss" betrieben wurde.

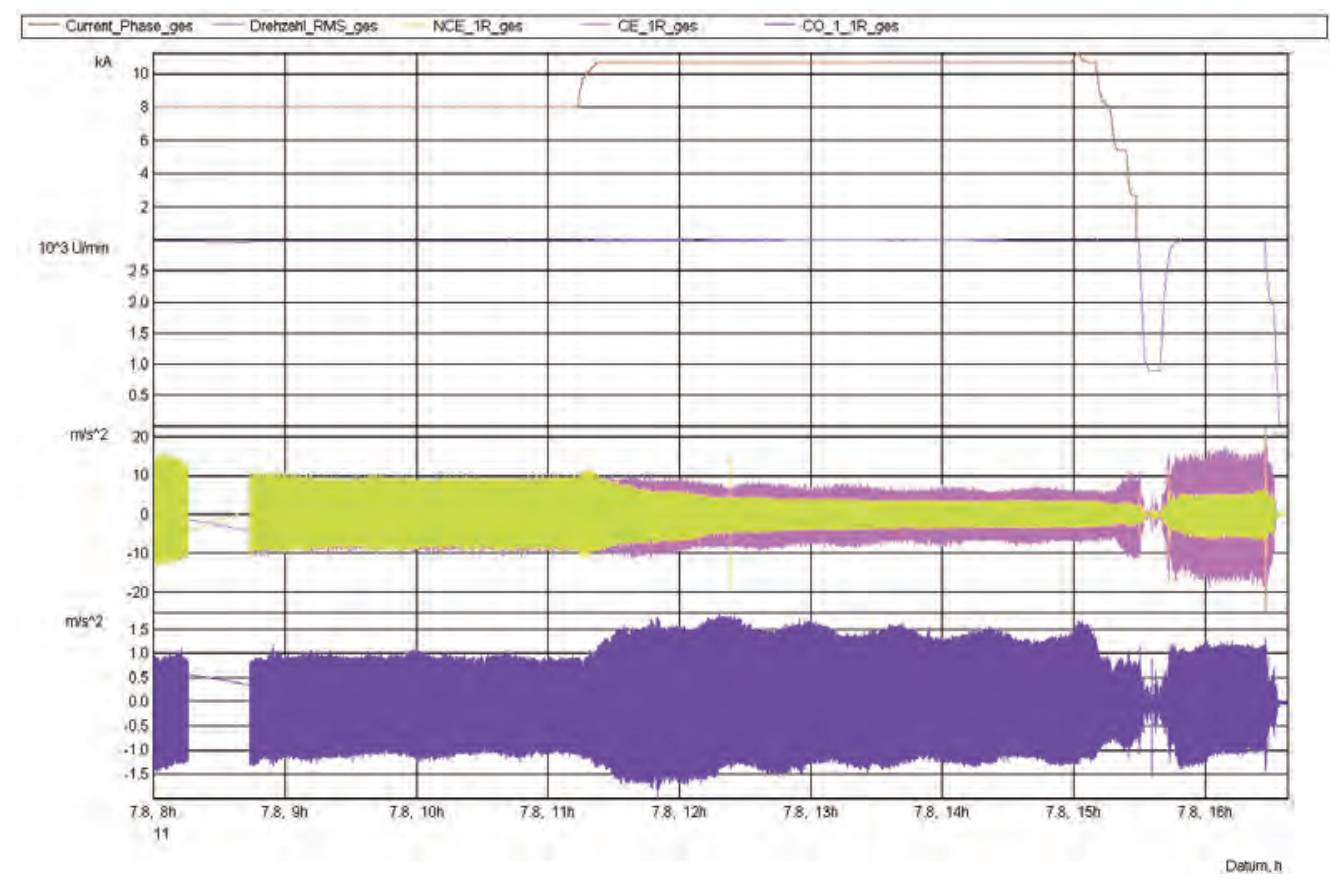

Bild 3.9: Fenster 1-Phasenstrom, Fenster 2-Drehzahl, Fenster 3- Schwingungen im Wickelkopfbereich, Fenster 4-Blechpaketschwingungen

Der Betriebsmodus „Klemmen-Kurzschluss“ ermöglicht durch die Korrelation von Schwingungen und Temperatur neben dem Monitoring der Kupfertemperatur eine eindeutige Analyse von verursachenden elektromagnetischen Effekten. Analysen in dieser Form ermöglichen Aussagen über das zukünftige Maschinenverhalten im realen Kraftwerksbetrieb.

\section{4) Optische Schwingungssensoren (FOA) bei Prototypentests}

Es hat sich gezeigt, dass faseroptische Sensoren geeignet sind, in einem Generator Messaufgaben zu übernehmen, da sie unempfindlich gegenüber den dort herrschenden sehr starken elektrischen und magnetischen Feldern sind. Eine Hauptaufgabe bestand in der Messung der Erwärmung der Generatorwicklung unter verschiedenen Betriebszuständen. Neben der Temperatur war aber auch das Schwingungsverhalten, speziell des sogenannten Wicklungskorbs, in den die Enden der Wicklung münden, von 
großem Interesse. Da dieser auf Hochspannungspotential liegt, kamen nur optische Beschleunigungsaufnehmer in Frage

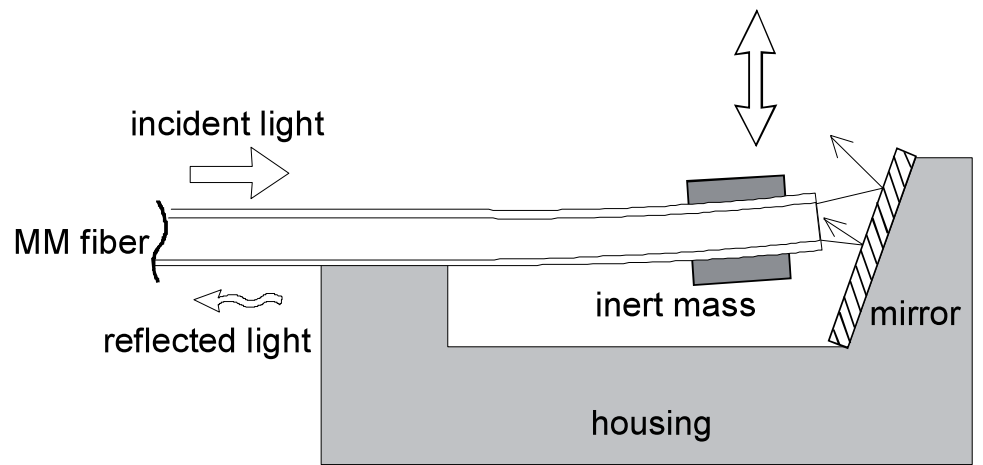

Bild 4.1: Optische Schwingungsmessung durch das Prinzip der Intensitätsmodulation

Es gibt zwar verschiedene kommerzielle Ausführungen, aber hat Siemens einen besonders einfachen Aufbau entwickelt, der hier zum Einsatz kam [3]. Er zeichnet sich durch einen sehr kompakten Sensorkopf aus, an dem eine Teflonader angekoppelt ist. Dieser basiert auf dem Prinzip des Faserfühlhebels mit Intensitätsmodulation. Bild 4.1 zeigt die Wirkungsweise schematisch auf. Die Sende-LED und die optoelektronische Wandlereinheit (Detektor) samt analoger Signalvorverarbeitung befinden sich auf einer Einschubplatine. Einen Rahmen mit der notwendigen Stromversorgung kann bis zu 16 Platinen, d. h. Kanäle aufnehmen. Dies reicht aus, um mit 16 Mess-Stellen die komplette Schwingungsanalyse eines Wicklungskorbs durchführen zu können. Diese technische Variante im Design der optischen Schwingungssensoren ermöglicht eine vereinfachte Implementierung der Sensoren in der Maschine und hat Vorteile bei möglichen Modifikationen der Generatorständerwicklung.

Für die modale Betriebsschwingungsanalyse speziell der potentialbeaufschlagten Generatorkomponenten bilden optische Sensoren basierend auf dem Prinzip der Intensitätsmodulation mittlerweile quasi einen Standard.

Bild 4.2 zeigt eine auf Messdaten beruhende ODS (operational-deflection-shape) Analyse von Generatorwickelkopf- und Blechpaketschwingungen in unterschiedlichen Maschinenzuständen.

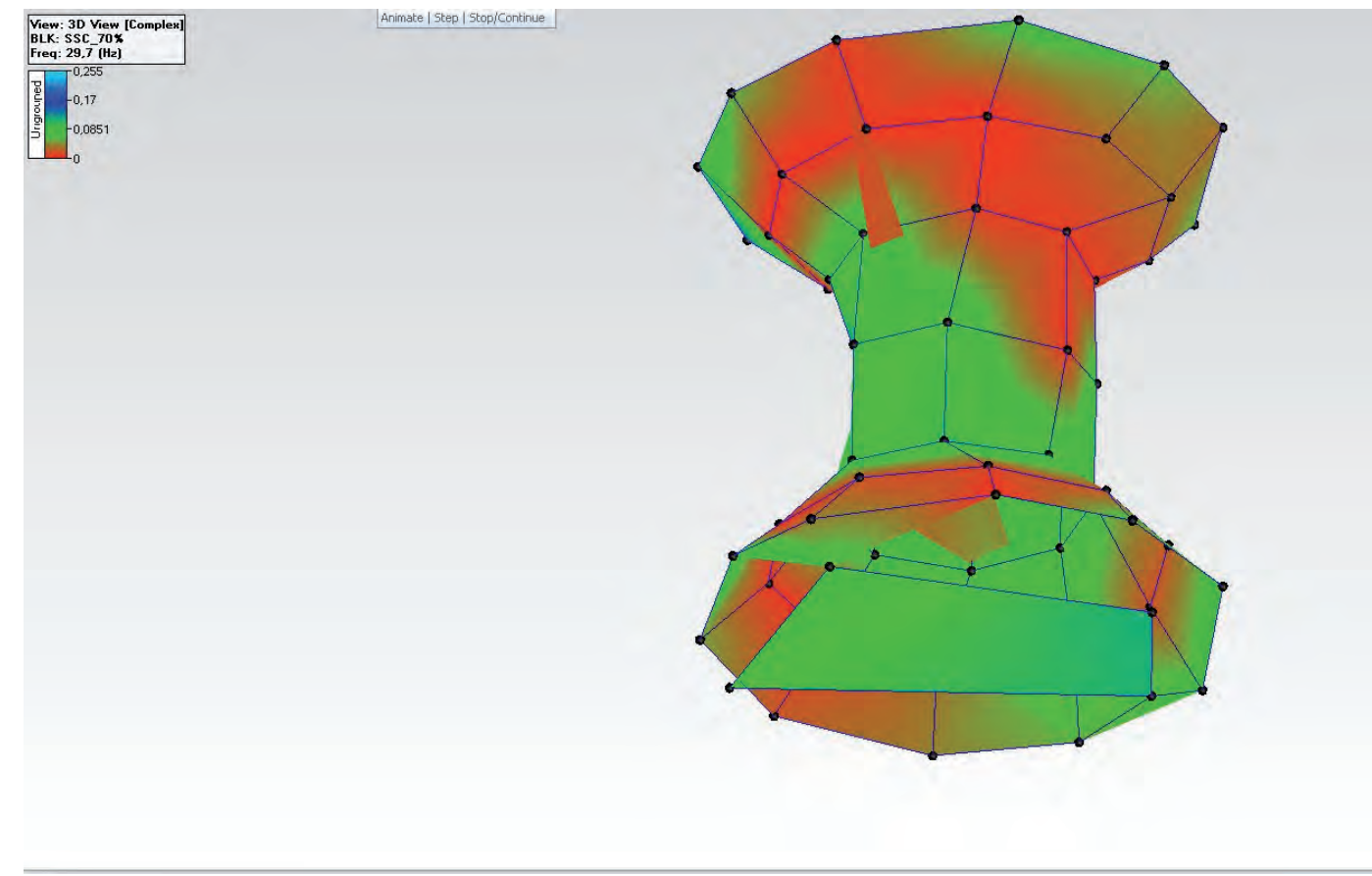

Bild 4.2: Modale Betriebsschwingungsanalyse, Blechpaket-Wickelkopf durch ICP- und FOA Sensoren 
Bei dieser ODS Analyse wurden die Schwingungssensoren durch gleichzeitige Datenaufnahme mit verschiedenen Temperaturen und Maschinenzuständen korreliert. Die Validierung der Berechnungsmodelle war somit möglich.

\section{5) Zusammenfassung}

Die Korrelation von Temperatur-, Schwingungs- sowie Maschinendaten lässt eine detaillierte Analyse des Maschinenverhaltens zu und hilft die Auslegungsmodelle des Designprozesses zu validieren. Dabei kommt der Temperaturerfassung der Ständerwicklung eine besondere Rolle zu. Der Einsatz optischer Temperatursensoren in Verbindung mit bewährten optischen Schwingungsaufnehmern ermöglicht die Validierung der neuen leistungsstarken luftgekühlten 370MVA Baureihe von Turbogeneratoren der Siemens AG. Die Entwicklung dieser Technologie zum Einbetten der Sensoren während des Fertigungsprozesses ermöglicht das Überwachen von Generatorständerwicklungen in Kraftwerken auch hinsichtlich der Verbesserung von Lebensdaueranalysen. In Verbindung mit anderen optischen Sensoren z.B. Schwingungsaufnehmern können verbesserte Analysen des Maschinenverhaltens bei verschiedenen Lastzuständen durchgeführt werden.

\section{6) Ausblick}

Die Bedeutung der Erfassung der Generatorkupferstabtemperatur wird im Rahmen der Änderung der Energieversorgung hin zu mehr dezentralen Erzeugungseinheiten an Bedeutung gewinnen. Die Vorraussetzung der „Smart-Grids“ sind „intelligente“ Netze aber auch „intelligentere“ Erzeugereinheiten. Somit kann es für die Kraftwerksbetreiber notwendig sein den jeweiligen Zustand ihrer Schlüsselkomponente Generator unter verschiedenen Betriebsbedingungen genau zu kennen. Optische Messverfahren spielen hier eine Schlüsselrolle. Die Anforderungen an die elektrischen und mechanischen Eigenschaften z.B. der Generatorständerwicklung würden sich im Rahmen des zukünftigen geplanten Energieversorgungsmixes ändern. Die optische Messtechniken bieten Potential das Maschinenverhalten zu jeglichem Zeitpunkt zu überwachen und zu analysieren.

Literatur

1. Kenichi hattori, Hiroshi Okabe, Kazumasa Ide, Keiji Kobashi, Takashi Watanabi, „Performance Evaluation and Measurement of the 250-MVA Class Air-Cooled Turbo Generator, Session 2004 (c) Cigre

2. S. Strack, R. Weidner, T. Bosselmann, M. Willsch, M. Villnow „Faseroptische Messtechnik zur Online-Überwachung und Diagnostik bei großen Generatoren in Kraftwerken „, ITG-Fachtagung Sensoren und Messsysteme, Nürnberg, Mai 2010

3. M. Villnow, M. Willsch, T. Bosselmann, B. Schmauss, „Novel fiber-optical accelerometer for monitoring tasks of power generators", ITG-Fachtagung Sensoren und Messsysteme, Nürnberg, Mai 2011

\section{Anschriften der Autoren}

Dipl.-Ing. Sebastian Strack, Siemens AG, Energy Sector, Fossil Power Generation, Generator Engineering, Abt. E F PR GN EN SE 22, D-45478 Mülheim a. d. Ruhr, Rheinstr. 100,

Tel.: 0049-208-456-5171, Fax: 0049-208-456-1847, e-mail: strack.sebastian@siemens.com

Dipl.-Ing. Jürgen Weidner, Siemens AG, Energy Sector, Fossil Power Generation, Generator Engineering, Abt. E F PR GN QM AE, D-45478 Mülheim a. d. Ruhr, Rheinstr. 100,

Tel.: 0049-208-456-4542, e-mail: juergen.r.weidner@siemens.com

Dr.-Ing. Thomas Bosselmann, Dipl.-Ing. Michael Villnow, Dr.-Ing. Michael Willsch, Siemens AG, Corporate Technology, Corporate Research and Technologies, Abt. CT T DE HW 3, D-91058 Erlangen, GüntherScharowsky-Str. 1, e-mail: thomas.bosselmann@siemens.com 